\title{
Association between the On-Plane Angular Motions of the Axle-Chain System and Clubhead Speed in Skilled Male Golfers
}

\author{
Morgan V. Madrid, Marco A. Avalos, Nicholas A. Levine, Noelle J. Tuttle, Kevin A. Becker \\ and Young-Hoo Kwon * \\ Biomechanics Laboratory, Texas Woman's University, Denton, TX 76204, USA; mmadrid1@twu.edu (M.V.M.); \\ mavalos1@twu.edu (M.A.A.); nlevine@twu.edu (N.A.L.); ntuttle@twu.edu (N.J.T.); kbecker1@twu.edu (K.A.B.) \\ * Correspondence: ykwon@twu.edu; Tel.: +1-940-898-2598
}

Received: 1 August 2020; Accepted: 18 August 2020; Published: 19 August 2020

check for updates

\begin{abstract}
The on-plane rotations of the inclined axle-chain system on the functional swing plane (FSP) can represent the angular motions of the golfer-club system closely. The purpose of this study was to identify key performance factors in golf through a comprehensive investigation of the association between the angular motion characteristics of the axle-chain system and clubhead speed in skilled golfers. Sixty-six male golfers (handicap $\leq 3$ ) performed full-effort shots in three club conditions: driver, 5-iron, and pitching wedge. Swing trials were captured with an optical motion capture system, and the hip/shoulder lines, upper lever, club, and wrist angular positions/velocities were calculated. Time, angular position, range of rotation, and peak angular velocity parameters were extracted and their correlation coefficients (Pearson and Spearman) to actual and normalized clubhead speeds were computed $(p<0.05)$. Higher clubhead speed was associated with shorter downswing phases, larger rotation ranges (hip/shoulder lines, and upper lever), larger hip-shoulder separation at impact, delayed transitions (hip line and upper lever), faster rotations (backswing, downswing, and impact), and larger angular velocity losses (hip line and upper lever) with additional club- and body-specific correlations. Clubhead speed was not well associated with wrist cock angles/ranges, $\mathrm{X}$-factors/stretches, and timings of the downswing peak.
\end{abstract}

Keywords: double pendulum; kinematic sequences; delayed release; transition phase

\section{Introduction}

The golf swing is an angular motion-dominant sport movement in which a high clubhead speed is generated primarily by the angular motions of the golfer-club system. To develop a high clubhead speed, the golfer must (1) generate large external moments by using the ground reaction forces (GRFs) and moments (GRMs), and (2) transfer angular momentum sufficiently from the body to the club [1]. To maximize the clubhead speed, it is imperative to not only use the ground effectively/efficiently, but also move the body/club in an orchestrated manner during the swing. The legs are primarily involved in the golfer-ground interaction, while the trunk/arms are responsible for generation of a fast-angular motion of the club. Han and colleagues [2] modeled the upper part of the golfer-club system (i.e., pelvis and above) as an axle-chain system consisting of an inclined multi-segment axle (trunk) and an open chain. The open chain was further modeled as a functional double-pendulum (FDP) system [3] in which the thorax, shoulder girdles, and arms form the upper lever (UL), while the club constitutes the lower lever (LL). In the FDP model, the hub of the open chain is located at the mid-section of the trunk through which the functional swing plane (FSP) [4] typically passes.

Effective central-to-peripheral (i.e., proximal-to-distal) angular momentum transfer that leads to a high clubhead speed can be achieved by well-orchestrated angular motions of the axle-chain system 
in which the kinematic sequences (i.e., backswing, transition, and downswing sequences) play key roles [2]. Interest in the kinematic sequence in golf has grown ever since it was initially investigated in striking and throwing motions [5] and subsequently in other sport motions [6-8]. The end outcome of good proximal-to-distal kinematic sequences is maximization of the speed of the distal end of the kinematic chain (i.e., clubhead) through effective summation of the segmental angular velocities of the elements of the axle-chain system [2]. A proximal-to-distal kinematic sequence typically comes with more efficient use of the muscles (i.e., stretch-shortening cycle (SSC)) along with minimization of the moment of inertia of the chain for each joint motion.

Summation of speed in golf has been established in the perspective of angular velocity peaks of the segments [9-13] or linear velocity peaks of the joints/segments [14-16]. Full or partial proximal-to-distal sequences of angular velocity peaks have been reported in protocols examining the difference between clubs [10], skill levels [9,13], and hip-shoulder torsional separation styles [2]. Early generation of pelvis rotation during the backswing and downswing phases [17] along with large ranges of hip [18] and shoulder [19] motions contribute to higher clubhead speed. The wrist joint, the most distal joint in the axle-chain system, has also been investigated for the role it plays in increasing clubhead speed. More wrist cock [20] and delayed onset of wrist motion (also known as delayed release) [21,22] have been associated with increased clubhead speed. The summation of speed principle also establishes that, as the motion sequence is nearing completion, the system follows a proximal-to-distal deceleration pattern with the proximal segments slowing down as the acceleration increases in the next distal segment $[9,16]$.

In addition to the backswing and downswing sequences based on the summation of speed principle, the backswing-to-downswing transition sequence also has received attention in the perspective of SSC $[9,23]$. Since the distal end of the axle-chain system is not constrained, a continuous backswing-to-downswing transition motion tends to lead to a proximal-to-distal transition sequence within the axle-chain system. A complete proximal-to-distal transition sequence can naturally induce SSC-style activation of the muscles that are responsible for generating angular motions of the axle-chain system. In a study involving a group of elite male golfers, Han and colleagues [2] identified four hip-shoulder separation styles and reported that the hip-shoulder separation pattern during the backswing influenced the backswing-to-downswing transition sequence, resulting in partial or full proximal-to-distal sequences.

Another group of swing concepts that are related to orchestrated behavior of the axle-chain system and have received substantial attention are the X-factor (i.e., hip-shoulder separation angle) and the X-factor stretch [24,25]. Investigators have compared X-factor parameters among different golfer groups or swing conditions and reported significant inter-group or inter-condition differences: e.g., skill groups [13,24,26,27], ball speed groups [28], genders [29], effort levels [30], and training stages [31]. While correlation/regression studies involving large heterogeneous samples have reported significant associations between X-factor parameters and club/ball speed [20,28], it is likely that these associations were practically driven by the heterogeneity in the samples. Correlation studies involving homogeneous golfer samples (e.g., skilled male golfers) in fact revealed no significant association between $X$-factor/stretch parameters and clubhead speed [2,3]. In elite male golfers, however, it was reported that the overall hip-shoulder separation pattern during the backswing did affect the backswing-to-downswing transition pattern/sequence [2].

While consistency in shot distance and direction is the most essential aspect of golf performance [4], the importance of shot distance itself increases with the level of competitiveness [3,9]. Shot distance is directly correlated to the clubhead speed at impact [21], and a golfer's ability to generate a high clubhead speed with sufficiently accurate direction control depends on well-orchestrated angular motions of the axle-chain system $[2,3,22]$ as well as the level of interaction with the ground [1]. Clubhead speed in fact can serve as an effective indicator of the quality of the swing mechanics employed in a golfer's swing. In order to truly identify the key angular motion characteristics that contribute to clubhead speed positively, however, it is crucial to eliminate the influence of the heterogeneity in the sample by 
employing a homogeneous group of skilled golfers instead of a heterogeneous sample of golfers of varying skill levels. The angular motions of the axle-chain system can be accurately represented by the planar angular motions of the body lines (hip line (HL) and shoulder line (SL)) and the levers (UL and LL) of the axle-chain system on the FSP [2]. The purpose of this study, therefore, was to identify key performance factors in golf swings through a comprehensive investigation of the association between the on-plane angular motion characteristics of the axle-chain system and clubhead speed in a group of elite male golfers. It was hypothesized that (1) temporal parameters, on-plane rotation ranges, and peak angular velocities of the axle-chain system would be significantly correlated to clubhead speed, and (2) proximal-to-distal kinematic sequences would be observed during the backswing, transition, and downswing in a group of skilled male golfers.

\section{Materials and Methods}

\subsection{Participants}

A total of 66 right-handed male skilled golfers (touring professionals, collegiate players, and teaching professionals; handicap $\leq 3)$ participated in this study. The average $( \pm$ SD) mass, height, age, and driving clubhead speed were $84.4 \pm 9.0 \mathrm{~kg}, 182.1 \pm 6.1 \mathrm{~cm}, 29.4 \pm 7.4$ years, and $49.4 \pm 2.1 \mathrm{~m} / \mathrm{s}(110.4 \pm 4.8 \mathrm{mph})$, respectively. The age range of the golfers was $19-50$ years. At the time of data collection, participants reported being free of any serious injuries that could affect their maximum-effort swing performance. Golfers who consistently presented outliers due to their unusual timing characteristics (e.g., extremely slow backswing) were excluded from the study. The study protocol was approved by Texas Woman's University's Institutional Review Board, and informed consent was obtained from each golfer prior to participation.

\subsection{Trial Conditions}

Participants performed maximal-effort swings until seven successful trials were collected in each of the following three club conditions: driver, 5-iron, and pitching wedge. Shots were performed indoors with foam balls against a wall (located $10.6 \mathrm{~m}$ away from the center of the ball mat and marked with a vertical target line) in a motion analysis laboratory. Success of a shot was determined by the shot characteristics, such as direction and launch angle judged from the ball impact location on the target wall, and golfer's perceived solidness of contact.

Each participant performed a self-selected warm-up prior to data collection and completed the trials with his own clubs/shoes. Trials were captured in the order of pitching wedge, 5-iron, and driver. Practice shots were allowed at the beginning of the data capture session and between the club conditions. The first five trials (out of the seven captured trials) that were free of marker labeling issues were used in further processing and analysis.

\subsection{Experimental Setup and Procedure}

Marker trajectories were captured by a 10-camera real-time optical motion capture system (Oqus, Qualisys, Gothenburg, Sweden) operated at $500 \mathrm{~Hz}$. Cameras were calibrated with a manufacturer-provided calibration wand before each data capture session. The ball mat (Hank Haney Profinity Practice Hitting Mat) was placed within the calibrated control volume. In the driver condition, rubber tees of various heights were used so that golfers could choose their preferred height.

Participants were required to be shirtless while wearing spandex shorts for minimization of the marker motion artifacts. Static trials (T-pose, ball mat, and club) were captured with seventy-six retro-reflective markers (spherical or hemispherical; $10-\mathrm{mm}$ in diameter) placed on the participant's body, shoes, club, ball, and the ball mat [2]. Once the static trials were captured, 15 static-only markers (4 clubface markers, the ball marker, and 10 body markers) were removed before proceeding with the swing trials. The T-pose static trial was captured once for each participant, while the ball mat static trial and the club static trial were captured once for each club condition. 
Captured trials were labeled by using Qualisys Tracking Manager (QTM, Qualisys, Gothenburg, Sweden). Labeled trials were saved in the c3d format (http://www.c3d.org) for subsequent processing.

\subsection{Data Reduction and Processing}

Captured marker coordinates were imported into Kwon3D Motion Analysis Suite (Version 5, Visol Inc., Seoul, Korea) for processing and analysis. Imported position data were upsampled to $1000 \mathrm{~Hz}$. A 4th-order zero-phase lag Butterworth low-pass filter was used in filtering the marker coordinates. The residual plot method outlined by Winter [32] (pp. 42-50) was used in assessing the frequency contents of the marker trajectory data, and markers were eventually clustered into three groups, each with different cutoff frequencies $(8,15$, and $30 \mathrm{~Hz})$ [2].

Secondary points (including joint centers) were defined and computed from the filtered marker coordinates and measured anthropometric parameters [2]. The Tylkowski-Andriacchi hybrid method was used in locating the hip joint centers, while the mid-point method was used in locating the shoulder joint centers $[4,33]$. The mid-trunk point was defined as the centroid of the xiphoid process and T12 (i.e., the spinous process of the 12th thoracic vertebra). The mid-hand point was defined as the centroid of the hand centers. The HL and the SL were defined by the vectors drawn from the left joint center to the right for the hips and shoulders, respectively. The UL was defined as the line drawn from the mid-trunk point to the mid-hand point, whereas the LL (club) was defined as the line drawn from the mid-hand point to the clubhead point.

\subsection{Variable Computation}

Twelve swing event frames were identified in each trial based on the club and lead arm (left arm) positions: BA (breakaway), MB (mid-backswing), LBA (late backswing, arm-based), LB (late backswing), EPR (end of pelvis rotation), TB (top of backswing), EDA (early downswing, arm-based), ED (early downswing), MD (mid downswing), BI (ball impact), MF (mid-follow-through), and MC (maximum cocking) [2]. BA is the instant the clubhead moves more than $3 \mathrm{~cm}$ away from the initial address position. EPR is the instant the direction of rotation of the pelvis changes from backward to downward. The ZYX (longitudinal-anteroposterior-mediolateral) rotation sequence was used in computing the orientation angles of the pelvis (rotation, lateral tilt, and anteroposterior tilt), and EPR was detected based on the first orientation angle. TB is the instant the club's direction of rotation changes from backward to downward in the frontal view. BI is the instant the clubface comes into contact with the ball. At the "mid" events, the club shaft is parallel to the ground in the frontal view. "Early" or "late" events are when the club shaft becomes vertical before or after the corresponding "mid" event. At the "arm-based" events, the lead arm is parallel to the ground. MC is the instant the wrist (the link between the upper lever and the club) is cocked the most on the FSP. BI was used as the zero-time event and all event times were expressed relative to BI. Five phases were defined from the events: backswing (BA-TB), downswing (TB-BI), transition (EPR-TB), early downswing (TB-EDA), and late downswing (EDA-BI).

The position and normal vector of the FSP were calculated from the clubhead trajectory (MD-MF) following the method outlined by Kwon et al. [4]. A group of on-plane angles of the axle-chain system (i.e., HL, SL, UL, club, and wrist cock) were defined on the FSP (Figure 1) as outlined by Han et al. [2], and ranges of rotation (ROR) and angular velocities were computed from the angular positions. The angle between the HL and the SL was used as the X-factor (Figure 1). The increases in the $X$-factor beyond EPR observed during the transition (EPR-TB) and the downswing (TB to maximum) were labeled as the backswing and downswing $X$-factor stretches, respectively. The total stretch was computed as the sum of the backswing and downswing stretches. 


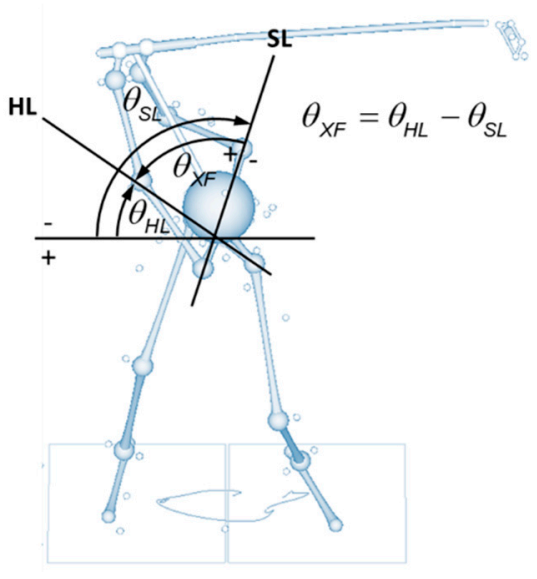

(a)

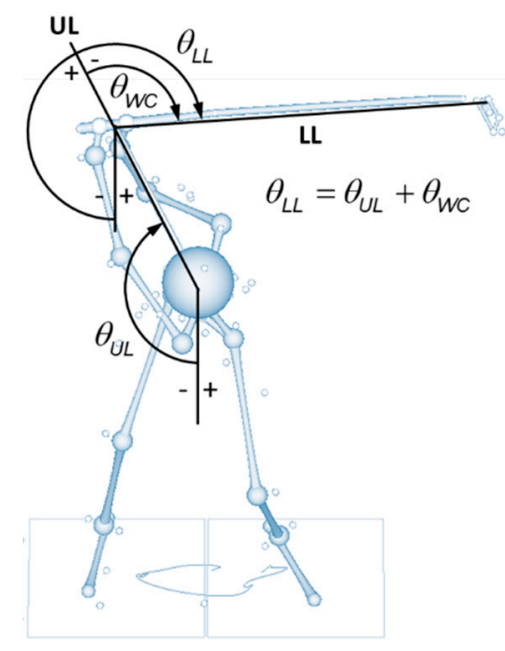

(b)

Figure 1. The on-plane angles of the axle-chain system defined on the functional swing plane. The hip line (HL) and shoulder line (SL) angles are relative to the horizontal line (a), while the upper lever (UL) and lower lever (LL; club) angles are relative to the vertical line (downward) (b). The wrist cock (WC) angle is the relative angular position of the LL to the UL. Counterclockwise angles yield positive values in these angles. The X-factor is the angle formed by the HL and the SL. A more backward-rotated (more negative) SL position relative to the HL yields a positive $X$-factor.

The instants the on-plane angular velocities of the body parts/club reached their respective peak values during backswing/downswing were identified as the peak points (Figure 2). The instants their on-plane rotations changed direction from backward to downward were identified as the transition points. All time data were expressed relative to BI. The actual clubhead speed (ACHS) was normalized to the body height $(\mathrm{BH})$ to obtain the normalized clubhead speed (NCHS).

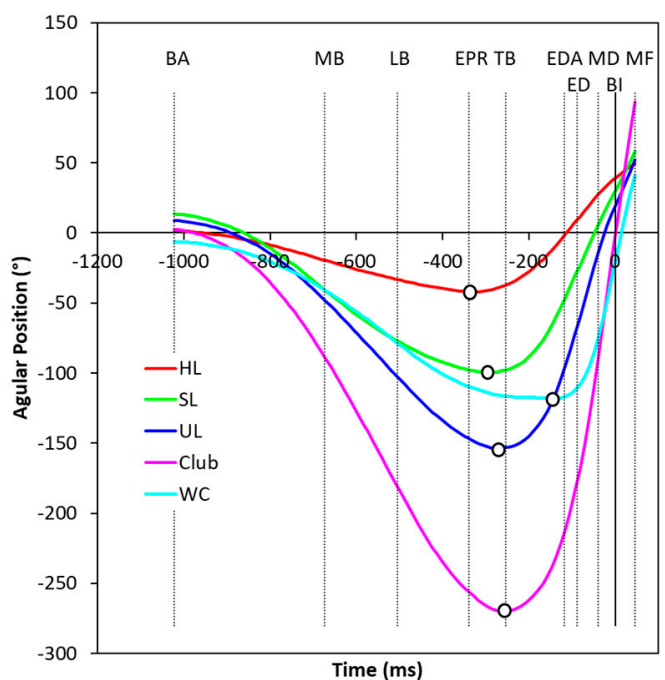

(a)

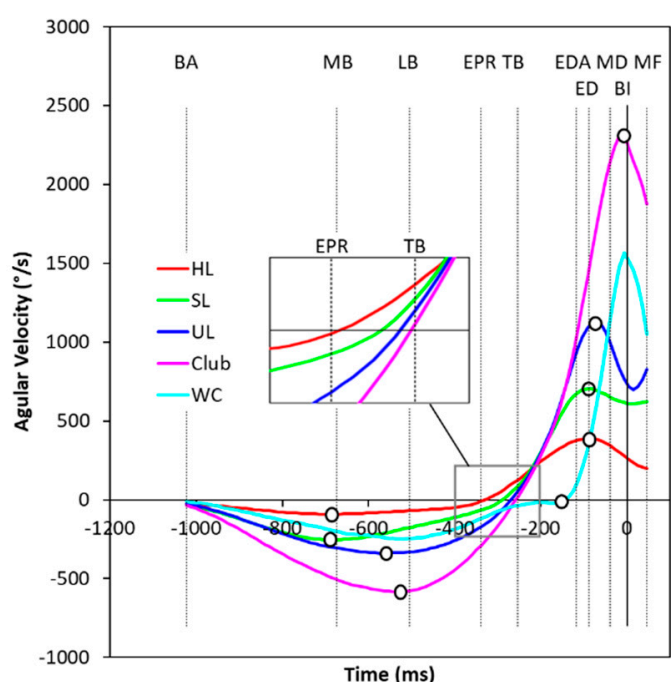

(b)

Figure 2. Ensemble-average on-plane angular position (a) and angular velocity (b) patterns of the axle-chain system (driver; $n=66$ ). Transition points and angular velocity peaks (backswing and downswing) are marked. Negative angular positions mean backward-rotated or cocked. Abbreviations: HL—hip line, SL—shoulder line, UL—upper lever, and WC—wrist cock angle. 
The following temporal/kinematic parameters were extracted from the trials for statistical analysis:

- $\quad$ Event times (relative to BI): BA, MB, LBA, LB, EPR, TB, EDA, ED, MD, and MC;

- Phase times: backswing (BA-TB), downswing (TB-BI), transition (EPR-TB), early downswing (TB-EDA), and late downswing (EDA-BI);

- Angular positions (address, transition, and BI) and RORs (backward and downward): UL, HL, and SL;

- Wrist cock angles (address, EDA, BI, and maximum) and ranges (cocking and uncocking);

- X-factors (address, EPR, TB, BI, and maximum) and range;

- $\quad \mathrm{X}$-factor stretches (backswing, downswing, and total);

- Times of backswing angular velocity peaks: HL, SL, UL, club, and wrist;

- Transition times: HL, SL, UL, club, and wrist;

- Times of downswing angular velocity peaks: HL, SL, and UL;

- Peak backswing angular velocities: $\mathrm{HL}, \mathrm{SL}, \mathrm{UL}, \mathrm{club}$, and wrist;

- Peak downswing angular velocities: HL, SL, UL, club, and wrist;

- Angular velocities at BI: HL, SL, and UL;

- Peak-to-BI angular velocity decreases: HL, SL, and UL.

\subsection{Statistical Analysis}

The average values of five repeated trials were used in the statistical analysis. The correlations between the select temporal/kinematic parameters and the clubhead speed (actual and normalized) were computed $(\alpha=0.05)$. Normality of the temporal/kinematic parameters was tested by using the Shapiro-Wilk test. When deviation from normality was caused by a small number (1 or 2) of outliers, the outliers were removed, and Pearson's correlation coefficient was calculated. When substantial deviation from normality was observed, Spearman's correlation coefficient was calculated instead. $r=0.1,0.3$, and 0.5 were used as the thresholds for small, moderate, and large correlations [34].

In addition, three two-way repeated-measure ANOVAs $(\alpha=0.017)$ were conducted for the kinematic-sequence time data (peak and transition points), one in each club condition, with two within-subject factors: SEQUENCE (backswing, transition, and downswing) and BODY (HL, SL, UL, and club). Post-hoc analysis was conducted if the BODY factor effect and/or the two-way interaction was significant. IBM SPSS Statistics (Version 25) was used for all statistical analyses.

\section{Results}

The average ACHS scores of the participants were $49.4 \pm 2.1 \mathrm{~m} / \mathrm{s}(110.4 \pm 4.8 \mathrm{mph}), 42.1 \pm 1.8 \mathrm{~m} / \mathrm{s}$ $(94.2 \pm 4.0 \mathrm{mph})$, and $38.2 \pm 1.9 \mathrm{~m} / \mathrm{s}(85.5 \pm 4.3 \mathrm{mph})$ for the driver, 5-iron, and pitching wedge, respectively. The average NCHS scores were $26.8 \pm 1.3 \mathrm{BH} / \mathrm{s}, 22.7 \pm 1.1 \mathrm{BH} / \mathrm{s}$, and $20.6 \pm 1.2 \mathrm{BH} / \mathrm{s}$, respectively. Tables $1-4$ present the average values of the temporal/kinematic parameters and their correlations to clubhead speeds (ACHS and NCHS) along with the counts of outliers removed.

\subsection{Event and Phase Times}

Among the swing events, the downswing events (TB, EDA, ED, and MD) showed significant $(p<0.05)$ correlations (moderate to large) to both ACHS and NCHS across all club conditions (Table 1 ). Among the backswing events, MB and LB were characterized by significant correlations (small to moderate) to ACHS in all club conditions with one exception (MB/driver). More delayed onset was associated with higher clubhead speed. Downswing phase times (total, early downswing, and late downswing) showed significant negative correlations (moderate to large) to NCHS across all club conditions (Table 1). The downswing and late downswing times also revealed significant correlations to ACHS in all club conditions. Shorter phase time was associated with higher clubhead speed. 
Table 1. Correlation coefficients of the event and phase times (in ms) to clubhead speed ( $n=66)$.

\begin{tabular}{|c|c|c|c|c|c|c|c|c|c|}
\hline \multirow{3}{*}{ Parameter } & \multicolumn{9}{|c|}{ Club } \\
\hline & \multicolumn{3}{|c|}{ Driver } & \multicolumn{3}{|c|}{ 5-Iron } & \multicolumn{3}{|c|}{ Pitching Wedge } \\
\hline & $M \pm S D$ & ACHS & NCHS & $M \pm S D$ & ACHS & NCHS & $M \pm S D$ & ACHS & NCHS \\
\hline \multicolumn{10}{|c|}{ Event time (relative to BI) } \\
\hline $\mathrm{BA}$ & $-1022 \pm 105$ & 0.226 & 0.172 & $-999 \pm 97$ & 0.223 & 0.221 & $-992 \pm 92$ & $0.401 *$ & $0.390 *$ \\
\hline MB & $-672 \pm 63$ & $0.248^{*}$ & 0.146 & $-670 \pm 59$ & 0.213 & 0.166 & $-672 \pm 58$ & $0.343 *$ & $0.307^{*}$ \\
\hline LBA & $-515 \pm 48$ & 0.162 & 0.121 & $-512 \pm 45$ & 0.126 & 0.136 & $-511 \pm 48$ & 0.218 & 0.234 \\
\hline LB & $-503 \pm 49$ & $0.287^{*}$ & 0.183 & $-493 \pm 44$ & $0.248^{*}$ & 0.184 & $-491 \pm 44$ & $0.316^{*}$ & $0.282 *$ \\
\hline EPR & $-336 \pm 25^{\#}$ & 0.177 & 0.239 & $-334 \pm 26^{\#}$ & 0.143 & 0.230 & $-338 \pm 26[1]$ & $0.258^{*}$ & 0.405 * \\
\hline ТВ & $-253 \pm 26$ & $0.481 *$ & $0.446^{*}$ & $-256 \pm 22[1]$ & $0.393 *$ & $0.405 *$ & $-266 \pm 25[1]$ & $0.441 *$ & $0.495 *$ \\
\hline EDA & $-118 \pm 9$ & $0.531 *$ & $0.475^{*}$ & $-113 \pm 9$ & $0.595 *$ & $0.612 *$ & $-117 \pm 13^{\#}$ & $0.720 *$ & $0.654^{*}$ \\
\hline ED & $-88 \pm 6$ & $0.810 *$ & $0.735 *$ & $-91 \pm 7$ & $0.736 *$ & $0.773 *$ & $-97 \pm 8[1]$ & $0.761 *$ & 0.811 * \\
\hline MD & $-39 \pm 2$ & $0.745^{*}$ & $0.644^{*}$ & $-37 \pm 2^{\#}$ & $0.737^{*}$ & $0.747^{*}$ & $-38 \pm 3$ & $0.802 *$ & $0.808^{*}$ \\
\hline $\mathrm{MC}$ & $-183 \pm 71^{\#}$ & 0.174 & 0.199 & $-183 \pm 75^{\#}$ & 0.161 & 0.232 & $-207 \pm 85^{\#}$ & 0.176 & 0.218 \\
\hline \multicolumn{10}{|c|}{ Phase time } \\
\hline BS & $769 \pm 100$ & -0.112 & -0.064 & $741 \pm 88$ & -0.125 & -0.108 & $724 \pm 78$ & $-0.284^{*}$ & -0.250 * \\
\hline DS & $253 \pm 26$ & $-0.481 *$ & $-0.446^{*}$ & $256 \pm 22[1]$ & $-0.393 *$ & $-0.405^{*}$ & $266 \pm 25[1]$ & $-0.441 *$ & -0.495 * \\
\hline Transition & $83 \pm 31$ & $0.274^{*}$ & 0.168 & $77 \pm 31$ & 0.224 & 0.126 & $72 \pm 30$ & 0.162 & 0.070 \\
\hline Early DS & $136 \pm 25$ & $-0.318^{*}$ & $-0.302 *$ & $144 \pm 23$ & -0.211 & $-0.261^{*}$ & $151 \pm 26$ & -0.199 & $-0.268^{*}$ \\
\hline Late DS & $118 \pm 9$ & -0.531 * & $-0.475^{*}$ & $113 \pm 9$ & $-0.595^{*}$ & $-0.612^{*}$ & $117 \pm 13^{\#}$ & -0.720 * & -0.654 * \\
\hline
\end{tabular}

Abbreviations: ACHS—actual clubhead speed, NCHS—normalized clubhead speed, BA—breakaway, MB—mid backswing, LBA—late backswing, arm-based, LB-late backswing, EPR—end of pelvis rotation, TB - top of backswing, EDA—early downswing, arm-based, ED—early downswing, MD—mid downswing, BI—ball impact, MC-maximum cocking, BS-backswing, and DS-downswing. \# Nonparametric (Spearman's) correlation coefficient; [ ] Number of outliers removed; * Statistically significant $(p<0.05)$.

\subsection{On-Plane Angular Positions and Ranges}

The transition (maximum backward-rotated) positions and RORs (backward and downward) of the HL, SL, and UL showed significant correlations (small to large) to NCHS across all club conditions (Table 2). The angular position at BI of the HL also showed significant correlations (moderate) to NCHS across all club conditions. The angular position at BI and downward ROR of the HL and the transition position and RORs (backward and downward) of the SL/UL revealed significant correlations (small to moderate) to ACHS. More backward-rotated transition position, more downward-rotated position at $\mathrm{BI}$, and larger RORs were associated with higher clubhead speed.

In the on-plane motion of the wrist, no consistent correlation profile was identified (Table 2). The wrist cock angle at EDA showed significant correlations (small to moderate) to clubhead speeds (ACHS and NCHS) in the driver condition only. More wrist cocking at EDA was associated with higher clubhead speed. Among the X-factors, the values at TB and BI were found to be significantly correlated (small to moderate) to ACHS across all club conditions (Table 2). The X-factor at BI was also correlated (moderate) to NCHS in two club conditions (iron and wedge). More HL-SL separations at these events were associated with higher clubhead speed. No consistent significant correlation was observed in the $\mathrm{X}$-factor stretches.

\subsection{Kinematic Sequence Time Parameters}

In terms of the time of backswing angular velocity peak, only the SL revealed significant correlations (small to moderate) to both ACHS and NCHS across all club conditions (Table 3). The UL also showed significant correlations (small) to ACHS in all club conditions except the pitching wedge. Delayed onset of the angular velocity peaks during the backswing was associated with higher clubhead speed. The times of transition of the HL, UL, and the club showed significant correlations (small to large) to NCHS across all club conditions. The UL and the club also showed significant correlations (moderate) to ACHS across all club conditions. In these cases, delayed transitions were associated with 
higher clubhead speed. The timings of angular velocity peaks in the downswing were characterized by a lack of significant correlation to clubhead speed.

Table 2. Correlation coefficients of the angular positions and ranges (in $\left.{ }^{\circ}\right)$ to clubhead speed $(n=66)$.

\begin{tabular}{|c|c|c|c|c|c|c|c|c|c|}
\hline \multirow{3}{*}{ Parameter } & \multicolumn{9}{|c|}{ Club } \\
\hline & \multicolumn{3}{|c|}{ Driver } & \multicolumn{3}{|c|}{ 5-Iron } & \multicolumn{3}{|c|}{ Pitching Wedge } \\
\hline & $M \pm S D$ & ACHS & NCHS & $M \pm S D$ & ACHS & NCHS & $M \pm S D$ & ACHS & NCHS \\
\hline \multicolumn{10}{|c|}{ Hip line angle } \\
\hline At address & $3 \pm 5$ & 0.158 & $0.269^{*}$ & $2 \pm 4$ & 0.171 & 0.204 & $2 \pm 4$ & 0.131 & 0.169 \\
\hline Transition & $-42 \pm 8$ & -0.010 & $-0.266^{*}$ & $-38 \pm 7$ & 0.046 & $-0.270 *$ & $-34 \pm 7$ & -0.189 & $-0.354^{*}$ \\
\hline At BI & $39 \pm 8$ & $0.325 *$ & $0.315^{*}$ & $31 \pm 7$ & $0.382 *$ & $0.373 *$ & $27 \pm 7$ & $0.422 *$ & $0.400^{*}$ \\
\hline Backward ROR & $45 \pm 8$ & 0.096 & $0.409 *$ & $40 \pm 7$ & 0.051 & $0.397^{*}$ & $37 \pm 7$ & $0.274 *$ & $0.470^{*}$ \\
\hline Downward ROR & $81 \pm 10$ & $0.278^{*}$ & $0.472 *$ & $69 \pm 9$ & $0.253^{*}$ & $0.495^{*}$ & $62 \pm 9$ & $0.469 *$ & $0.584^{*}$ \\
\hline \multicolumn{10}{|c|}{ Shoulder line angle } \\
\hline At address & $15 \pm 5$ & 0.233 & $0.300 *$ & $12 \pm 4$ & 0.080 & 0.213 & $12 \pm 4$ & 0.070 & 0.206 \\
\hline Transition & $-100 \pm 8$ & $-0.254^{*}$ & $-0.339 *$ & $-96 \pm 9$ & -0.161 & $-0.338^{*}$ & $-93 \pm 9[1]$ & $-0.362 *$ & $-0.374^{*}$ \\
\hline At BI & $30 \pm 5$ & 0.180 & 0.192 & $23 \pm 4^{\#}$ & 0.173 & 0.126 & $21 \pm 3[1]$ & 0.096 & 0.134 \\
\hline Backward ROR & $115 \pm 11$ & $0.287^{*}$ & $0.422 *$ & $108 \pm 10$ & 0.173 & $0.379 *$ & $104 \pm 10$ & 0.351 * & $0.444^{*}$ \\
\hline Downward ROR & $130 \pm 9$ & $0.306^{*}$ & 0.439 * & $119 \pm 9$ & 0.241 & $0.412 *$ & $114 \pm 9$ & $0.403 *$ & $0.454^{*}$ \\
\hline \multicolumn{10}{|c|}{ Upper lever angle } \\
\hline At address & $9 \pm 4$ & $0.280 *$ & 0.260 * & $6 \pm 3$ & 0.020 & 0.171 & $5 \pm 1$ & 0.055 & 0.206 \\
\hline Transition & $-154 \pm 9$ & $-0.333^{*}$ & $-0.394^{*}$ & $-148 \pm 8$ & $-0.335^{*}$ & $-0.400 *$ & $-142 \pm 9$ & $-0.398^{*}$ & -0.410 * \\
\hline At BI & $19 \pm 5$ & -0.064 & 0.015 & $12 \pm 4$ & -0.193 & -0.025 & $11 \pm 4$ & $-0.276^{*}$ & -0.139 \\
\hline Backward ROR & $164 \pm 10$ & $0.386^{*}$ & $0.437^{*}$ & $154 \pm 9$ & $0.312 *$ & $0.420 *$ & $147 \pm 10$ & $0.387^{*}$ & $0.443 *$ \\
\hline Downward ROR & $173 \pm 9$ & $0.299 *$ & $0.408^{*}$ & $160 \pm 8$ & $0.262 *$ & $0.414^{*}$ & $152 \pm 8$ & $0.316^{*}$ & $0.395 *$ \\
\hline \multicolumn{10}{|c|}{ Wrist cock angle } \\
\hline At address & $-6 \pm 4$ & -0.133 & -0.175 & $-9 \pm 4$ & -0.091 & -0.157 & $-10 \pm 4$ & -0.147 & -0.210 \\
\hline Max. cocking & $-121 \pm 10$ & -0.172 & -0.101 & $-114 \pm 8[1]$ & -0.078 & 0.030 & $-110 \pm 8[1]$ & -0.066 & -0.018 \\
\hline At BI & $-20 \pm 5$ & 0.204 & 0.068 & $-20 \pm 5$ & 0.135 & 0.026 & $-20 \pm 5$ & 0.185 & 0.112 \\
\hline At EDA & $-117 \pm 9^{\#}$ & $-0.362 *$ & $-0.265^{*}$ & $-111 \pm 8$ & -0.133 & -0.188 & $-105 \pm 7[1]$ & -0.109 & -0.131 \\
\hline Cocking range & $115 \pm 11$ & 0.104 & -0.019 & $106 \pm 10$ & 0.006 & -0.030 & $100 \pm 10$ & -0.029 & -0.031 \\
\hline Uncocking range & $101 \pm 11$ & $0.255^{*}$ & 0.119 & $95 \pm 10$ & 0.105 & 0.049 & $90 \pm 10$ & 0.119 & 0.111 \\
\hline \multicolumn{10}{|c|}{$X$-factor } \\
\hline At address & $-12 \pm 6$ & -0.067 & -0.036 & $-10 \pm 5$ & 0.072 & 0.004 & $-10 \pm 5$ & 0.042 & -0.028 \\
\hline At EPR & $56 \pm 8$ & 0.237 & 0.156 & $56 \pm 8$ & 0.228 & 0.169 & $56 \pm 8$ & $0.258^{*}$ & 0.199 \\
\hline At TB & $61 \pm 9$ & $0.265 *$ & 0.175 & $60 \pm 8$ & $0.247^{*}$ & 0.160 & $59 \pm 8$ & 0.270 * & 0.183 \\
\hline Maximum & $62 \pm 8$ & 0.218 & 0.159 & $61 \pm 8$ & 0.219 & 0.151 & $60 \pm 8$ & $0.263 *$ & 0.182 \\
\hline At BI & $9 \pm 8$ & $0.249 *$ & 0.235 & $8 \pm 7$ & $0.295^{*}$ & $0.309 *$ & $6 \pm 7$ & 0.391 * & $0.372 *$ \\
\hline Range & $74 \pm 9^{\#}$ & $0.306^{*}$ & 0.121 & $71 \pm 8^{\#}$ & 0.164 & 0.142 & $69 \pm 8$ & 0.226 & 0.192 \\
\hline \multicolumn{10}{|c|}{$X$-factor stretch } \\
\hline Backswing & $5 \pm 3^{\#}$ & 0.070 & 0.020 & $4 \pm 3^{\#}$ & 0.066 & -0.015 & $3 \pm 3^{\#}$ & 0.110 & 0.068 \\
\hline Downswing & $1 \pm 2^{\#}$ & $-0.258^{*}$ & -0.063 & $1 \pm 1^{\#}$ & -0.193 & -0.060 & $1 \pm 1^{\#}$ & -0.009 & 0.025 \\
\hline Total & $6 \pm 4^{\#}$ & -0.130 & -0.066 & $5 \pm 3^{\#}$ & -0.017 & -0.038 & $3 \pm 3^{\#}$ & 0.038 & 0.026 \\
\hline
\end{tabular}

Abbreviations: ACHS-actual clubhead speed, NCHS-normalized clubhead speed, ROR—range of rotation, EPR—end of pelvis rotation, TB—top of backswing, EDA—early downswing, and BI-ball impact. \# Nonparametric (Spearman's) correlation coefficient; [ ] Number of outliers removed; * Statistically significant $(p<0.05)$. 
Table 3. Correlation coefficients of the kinematic-sequence time parameters (in ms; relative to BI) to clubhead speed $(n=66)$.

\begin{tabular}{|c|c|c|c|c|c|c|c|c|c|}
\hline \multirow{3}{*}{ Parameter } & \multicolumn{9}{|c|}{ Club } \\
\hline & \multicolumn{3}{|c|}{ Driver } & \multicolumn{3}{|c|}{ 5-Iron } & \multicolumn{3}{|c|}{ Pitching Wedge } \\
\hline & $M \pm S D$ & ACHS & NCHS & $M \pm S D$ & ACHS & NCHS & $M \pm S D$ & ACHS & NCHS \\
\hline \multicolumn{10}{|c|}{ Backswing peak } \\
\hline Hip line & $-657 \pm 98^{\#}$ & 0.065 & 0.084 & $-646 \pm 94$ & 0.093 & 0.132 & $-644 \pm 93$ & 0.184 & 0.232 \\
\hline Shoulder line & $-675 \pm 75$ & $0.366^{*}$ & $0.249 *$ & $-665 \pm 75$ & $0.299 *$ & $0.272 *$ & $-668 \pm 77$ & $0.350 *$ & $0.337^{*}$ \\
\hline Upper lever & $-572 \pm 64^{\#}$ & $0.281 *$ & 0.214 & $-565 \pm 61$ \# & $0.273 *$ & 0.168 & $-565 \pm 57[1]$ & 0.155 & 0.099 \\
\hline Club & $-536 \pm 71$ & $0.243 *$ & 0.100 & $-553 \pm 72$ & 0.194 & 0.089 & $-569 \pm 74$ & 0.152 & 0.112 \\
\hline Wrist (cocking) & $-539 \pm 93$ & 0.217 & 0.063 & $-570 \pm 105$ & 0.169 & 0.068 & $-584 \pm 100$ & 0.140 & 0.071 \\
\hline Time difference & \multicolumn{3}{|c|}{$\mathrm{SL} / \mathrm{HL} \rightarrow \mathrm{UL} \rightarrow$ club } & \multicolumn{3}{|c|}{$\mathrm{SL} / \mathrm{HL} \rightarrow \mathrm{UL} /$ club } & \multicolumn{3}{|c|}{$\mathrm{SL} / \mathrm{HL} \rightarrow \mathrm{club} / \mathrm{UL}$} \\
\hline \multicolumn{10}{|c|}{ Transition } \\
\hline Hip line & $-327 \pm 24^{\#}$ & 0.215 & $0.294^{*}$ & $-321 \pm 25^{\#}$ & 0.211 & $0.318^{*}$ & $-323 \pm 29^{\#}$ & $0.315^{*}$ & $0.418^{*}$ \\
\hline Shoulder line & $-286 \pm 20^{\#}$ & 0.117 & 0.129 & $-288 \pm 25^{\#}$ & 0.121 & 0.086 & $-294 \pm 29^{\#}$ & $0.280^{*}$ & $0.326^{*}$ \\
\hline Upper lever & $-266 \pm 19$ & $0.442 *$ & $0.419 *$ & $-265 \pm 15[1]$ & $0.386^{*}$ & $0.382 *$ & $-273 \pm 19[1]$ & $0.479 *$ & $0.514^{*}$ \\
\hline Club & $-258 \pm 25$ & $0.499 *$ & $0.420 *$ & $-262 \pm 23$ & $0.429 *$ & $0.464 *$ & $-270 \pm 24[1]$ & $0.416^{*}$ & $0.447^{*}$ \\
\hline Wrist (uncocking) & $-164 \pm 63^{\#}$ & 0.182 & 0.206 & $-160 \pm 65^{\#}$ & 0.217 & $0.277^{*}$ & $-172 \pm 71^{\#}$ & $0.288^{*}$ & $0.330 *$ \\
\hline Time difference & \multicolumn{3}{|c|}{$\mathrm{HL} \rightarrow \mathrm{SL} \rightarrow \mathrm{UL} \rightarrow$ club } & \multicolumn{3}{|c|}{$\mathrm{HL} \rightarrow \mathrm{SL} \rightarrow \mathrm{UL} \rightarrow$ club } & \multicolumn{3}{|c|}{$\mathrm{HL} \rightarrow \mathrm{SL} \rightarrow \mathrm{UL} /$ club } \\
\hline \multicolumn{10}{|c|}{ Downswing peak } \\
\hline Hip line & $-87 \pm 18$ & -0.014 & -0.104 & $-76 \pm 23^{\#}$ & -0.037 & -0.146 & $-66 \pm 27^{\#}$ & -0.202 & $-0.306^{*}$ \\
\hline Shoulder line & $-84 \pm 25^{\#}$ & 0.184 & 0.082 & $-83 \pm 20^{\#}$ & 0.143 & 0.067 & $-75 \pm 28^{\#}$ & -0.006 & -0.046 \\
\hline Upper lever & $-73 \pm 6$ & 0.083 & 0.046 & $-70 \pm 6$ & 0.140 & 0.060 & $-68 \pm 6$ & 0.177 & 0.069 \\
\hline Time difference & \multicolumn{3}{|c|}{$\mathrm{HL} / \mathrm{SL} \rightarrow \mathrm{UL} \rightarrow$ club } & & \multicolumn{2}{|c|}{$\begin{aligned} \mathrm{SL} \rightarrow \mathrm{UL} & \rightarrow \text { club } \\
\mathrm{HL} & \rightarrow \text { club }\end{aligned}$} & \multicolumn{3}{|c|}{$\mathrm{SL} / \mathrm{UL} / \mathrm{HL} \rightarrow$ club } \\
\hline
\end{tabular}

Abbreviations: ACHS—actual clubhead speed, NCHS—normalized clubhead speed, and BI—ball impact. \# Nonparametric (Spearman's) correlation coefficient; [ ] Number of outliers removed; * Statistically significant $(p<0.05)$

The two-way repeated ANOVAs of the kinematic-sequence time parameters revealed significant SEQUENCE*BODY interactions in all club conditions: driver (Huynh-Feldt $F=44.384, p<0.001$, partial $\eta=0.406$ ), 5-iron (Huynh-Feldt $F=39.744, p<0.001$, partial $\eta=0.383$ ), and pitching wedge (Huynh-Feldt $F=37.251, p<0.001$, partial $\eta=0.368$ ). The most consistent proximal-to-distal sequence was observed in the transition phase (Table 3). Both the driver and 5-iron conditions showed full proximal-to-distal sequences, while the pitching wedge condition showed a partial sequence with the UL and club transitions not significantly separated.

In the backswing sequence, the timings of the HL and SL angular velocity peaks were not significantly separated with the tendency of the SL peak appearing earlier than the HL peak (Table 3; Figure $2 b$ ). The timing of the UL peak was significantly separated from the club peak only in the driver condition. The SDs of the backswing angular velocity peak times were substantially larger than those of the other sequences. In the downswing sequence, however, only the separation between the club and the rest of the axle-chain system (SL/HL/UL) was notable, which was consistent across all club conditions (Table 3; Figure 2b). The downswing angular velocity peaks of the body parts occurred in a narrow time window with inconsistent sequences, although some statistically significant separations were observed (e.g., SL vs. UL in the driver and 5-iron conditions).

\subsection{Angular Velocity Parameters}

The peak backswing angular velocities of the HL, SL, and UL were characterized by significant correlations (small to large, mostly moderate) to both ACHS and NCHS across all club conditions (Table 4). Faster backward rotations were associated with higher clubhead speed. All peak downswing angular velocities of the axle-chain system were significantly correlated to ACHS and NCHS across all club conditions. Faster downward rotations were associated with higher clubhead speed. At impact, 
the angular velocities of the SL and UL were significantly correlated (small to moderate) to both ACHS and NCHS with one exception (UL/driver vs. NCHS). Faster rotations at impact were associated with higher clubhead speed. The peak-to-BI angular velocity decrease in the HL and UL was significantly correlated (small to large) to NCHS across all club conditions. In addition, the HL also revealed significant correlations (moderate to large) to ACHS. More losses in the angular velocity were associated with higher clubhead speed.

Table 4. Correlation coefficients of the angular velocity parameters (in $\%$ s) to clubhead speed $(n=66)$.

\begin{tabular}{|c|c|c|c|c|c|c|c|c|c|}
\hline \multirow{3}{*}{ Parameter } & \multicolumn{9}{|c|}{ Club } \\
\hline & \multicolumn{3}{|c|}{ Driver } & \multicolumn{3}{|c|}{ 5-Iron } & \multicolumn{3}{|c|}{ Pitching Wedge } \\
\hline & $M \pm S D$ & ACHS & NCHS & $M \pm S D$ & ACHS & NCHS & $M \pm S D$ & ACHS & NCHS \\
\hline \multicolumn{10}{|c|}{ Backswing peak } \\
\hline Hip line & $-96 \pm 22[1]$ & $-0.314^{*}$ & $-0.435^{*}$ & $-86 \pm 21$ & $-0.275^{*}$ & $-0.451 *$ & $-79 \pm 19$ & $-0.464 *$ & -0.539 * \\
\hline Shoulder line & $-259 \pm 56$ & -0.332 * & $-0.311^{*}$ & $-247 \pm 53$ & -0.263 * & -0.334 * & $-239 \pm 51$ & $-0.401 *$ & -0.424 * \\
\hline Upper lever & $-349 \pm 47$ & $-0.330 *$ & $-0.308^{*}$ & $-337 \pm 44$ & $-0.322 *$ & $-0.341 *$ & $-329 \pm 43$ & $-0.409 *$ & $-0.413 *$ \\
\hline Club & $-610 \pm 87$ & -0.186 & -0.128 & $-576 \pm 83$ & -0.240 & -0.183 & $-568 \pm 77$ & $-0.296^{*}$ & -0.237 \\
\hline Wrist cocking & $-289 \pm 65^{\#}$ & 0.003 & 0.068 & $-268 \pm 62 \#$ & -0.070 & -0.012 & $-257 \pm 59$ & -0.097 & -0.055 \\
\hline \multicolumn{10}{|c|}{ Downswing peak } \\
\hline Hip line & $394 \pm 62$ & $0.447^{*}$ & $0.583 *$ & $340 \pm 61$ & $0.398 *$ & $0.579 *$ & $305 \pm 63$ & 0.541 * & $0.620 *$ \\
\hline Shoulder line & $711 \pm 63$ & 0.461 * & $0.580 *$ & $662 \pm 60$ & $0.398 *$ & $0.603 *$ & $622 \pm 63$ & 0.618 * & $0.716^{*}$ \\
\hline Upper lever & $1142 \pm 67$ & $0.552 *$ & $0.696^{*}$ & $1077 \pm 69$ & $0.546^{*}$ & $0.756^{*}$ & $1020 \pm 72[1]$ & $0.685 *$ & $0.802 *$ \\
\hline Club & $2364 \pm 115$ & $0.906^{*}$ & $0.787^{*}$ & $2341 \pm 108$ & $0.846^{*}$ & $0.799 *$ & $2251 \pm 122$ & $0.874 *$ & $0.867^{*}$ \\
\hline Wrist uncocking & $1615 \pm 138$ & $0.539 *$ & $0.506 *$ & $1627 \pm 127$ & $0.419 *$ & $0.465 *$ & $1563 \pm 131$ & $0.494 *$ & $0.558 *$ \\
\hline \multicolumn{10}{|c|}{ At BI } \\
\hline Hip line & $262 \pm 63$ & -0.150 & -0.115 & $259 \pm 53$ & -0.026 & 0.030 & $247 \pm 51$ & 0.155 & 0.185 \\
\hline Shoulder line & $610 \pm 65$ & $0.265^{*}$ & $0.299 *$ & $572 \pm 62$ & $0.258 *$ & $0.290 *$ & $553 \pm 63$ & $0.365 *$ & $0.383 *$ \\
\hline Upper lever & $725 \pm 84$ & $0.324 *$ & 0.220 & $705 \pm 80$ & $0.441 *$ & $0.315^{*}$ & $680 \pm 79$ & $0.494 *$ & $0.395 *$ \\
\hline \multicolumn{10}{|c|}{ Peak-to-BI decrease } \\
\hline Hip line & $-123 \pm 66[2]$ & $-0.476^{*}$ & $-0.552 *$ & $-81 \pm 62^{\#}$ & $-0.417^{*}$ & $-0.507^{*}$ & $-58 \pm 55^{\#}$ & $-0.517^{*}$ & -0.575 * \\
\hline Shoulder line & $-101 \pm 80^{\#}$ & -0.148 & -0.182 & $-89 \pm 71^{\#}$ & -0.083 & -0.150 & $-69 \pm 65^{\#}$ & -0.212 & -0.283 * \\
\hline Upper lever & $-417 \pm 100$ & -0.102 & $-0.287^{*}$ & $-372 \pm 94$ & -0.026 & $-0.285 *$ & $-336 \pm 91^{\#}$ & -0.183 & $-0.373 *$ \\
\hline
\end{tabular}

Abbreviations: ACHS-actual clubhead speed, and NCHS—normalized clubhead speed. \# Nonparametric

(Spearman's) correlation coefficient; [ ] Number of outliers removed; * Statistically significant $(p<0.05)$.

\section{Discussion}

The main purpose of this study was to investigate the association between the on-plane angular motion parameters of the axle-chain system and clubhead speed in three different club conditions. The on-plane angular positions, RORs, and angular velocities of the HL, SL, UL, club, and wrist were calculated along with a host of temporal parameters (event times and peak/transition times). The clubhead speed was normalized to the body height and the temporal/kinematic parameters were correlated to both the actual (ACHS) and normalized (NCHS) clubhead speeds. The coefficients of variation (COVs) of the ACHS were 4.3\%, 4.2\%, and 5.0\% for driver, 5-iron, and pitching wedge, respectively, while those of the NCHS were $4.9 \%, 4.8 \%$, and $5.9 \%$. The NCHS and the pitching wedge condition tended to show larger COVs than the ACHS and the driver/5-iron conditions, respectively.

The backswing-to-downswing time ratio was approximately 3.0 to 1 in the driver condition with a decreasing trend in the shorter clubs ( 2.9 to 1 for the 5-iron and 2.7 to 1 for the pitching wedge). As the club got shorter, the backswing time decreased while the downswing time increased (Table 1). The association between NCHS and event/phase times was expected, as faster motion naturally leads to shorter phases and delayed onset of events closer to BI (Table 1). Higher NCHS was associated with shorter downswing phase times (total, early downswing, and late downswing). One noteworthy finding was that the NCHS was associated with the downswing event times but not with the backswing event times. The timing of EPR, the beginning of the transition phase, showed significant deviations 
from normal distribution in the longer club conditions (Table 1). This suggests that the way golfers start the transition phase is highly dependent on the individual and the swing style employed.

In a study involving 74 male elite golfers, Han and colleagues [2] identified five distinct swing styles based on the X-factor pattern and the SL acceleration characteristic. The LBS (large backswing stretch) style, characterized by the longest transition phase and a complete (and well-spaced) proximal-to-distal transition sequence, showed an almost 1:1:1 (109:117:119 ms) time ratio between the transition, early downswing, and late downswing phases. The LDS (large downswing stretch) style scored an average time ratio of 1:2.5:2 (63:153:119 $\mathrm{ms}$ ) with the transition phase being substantially shorter than the early downswing phase. Simultaneous transitions of the SL, UL, and the club were observed in the LDS style. The average phase times in the current study $(83 \mathrm{~ms}, 136 \mathrm{~ms}$, and $118 \mathrm{~ms}$ for the transition, early downswing, and late downswing phases, respectively) were similar to those of the LSA (late shoulder acceleration) style ( $82 \mathrm{~m}, 137 \mathrm{~m}$, and $119 \mathrm{~ms}$, respectively) reported by Han and associates [2]. Further explanatory analysis revealed significant negative correlations between the phase times of the early downswing phase and the transition phase $(r=-0.584,-0.571$, and -0.526 for driver, 5 -iron, and pitching wedge, respectively), which means a longer transition phase is associated with a shorter early downswing phase. The findings of the study by Han and colleagues [2] and the current study collectively imply that the swing mechanics employed in the transition phase can be a key determinant of the biomechanical robustness of a golf swing. Therefore, more in-depth investigations on various transition strategies employed by skilled golfers are warranted.

The pitching wedge condition was characterized by additional significant correlations between the NCHS and the backswing event/phase times. Higher clubhead speed was associated with a shorter backswing phase and delayed onset of the backswing events. This suggests that the swing mechanics used in the pitching wedge condition could be somewhat different from that used in the longer club conditions. More in-depth investigations of the mechanics involved in full and partial wedge swings are necessary.

Higher NCHS was associated with larger on-plane angular motions (i.e., more backward-rotated transition positions and larger backward/downward RORs) of the HL, SL, and UL (Table 2; Figure 2a). Additional body-specific (e.g., HL angular position at BI) and club-specific (e.g., driver at address) significant correlations were observed in the factors contributing to larger RORs. The driver-specific additional correlations at address appeared to be related to the ball position, as the ball is typically located closer to the lead foot in the driver condition when compared to other club conditions. The HL-specific additional correlations also suggest that the pelvis position at impact can be useful in assessing the golfer's impact posture.

In general, the clubhead speed was not well associated with the wrist-cock angles/ranges (Table 2). Club-specific correlations of wrist-cock angle to clubhead speed, however, were observed in the driver condition at EDA. Unlike iron/wedge swings, one of the main goals in a driver swing is to maximize the distance. The GRF moment generated by the combined GRF about the center of mass (COM) in the frontal plane and the pivoting moment generated by individual foot GRFs about the combined center of pressure (COP) in the horizontal plane typically reach their peak values at or slightly before EDA [1]. The relative position of the club to the COM at EDA directly affects the moment of inertia of the golfer-club system and the resulting angular acceleration caused by the golfer-ground interaction moments. For a faster angular acceleration of the body-club system, therefore, it is imperative to keep the club closer to the body by maintaining the lag (relatively large wrist-cock angle) near EDA.

It is noteworthy that the X-factor/stretch parameters overall lack association to clubhead speed while the angular position/range and velocity parameters of the HL and the SL individually do exhibit significant associations. Among the $\mathrm{X}$-factors at various time points, only that at $\mathrm{BI}$ showed significant correlations (in 5-iron and pitching wedge). Higher NCHS was associated with larger HL-SL torsional separation at BI (i.e., more downward-rotated HL position than the SL). Promotion of the pelvis lateral tilt with the left hip positioned higher at BI and suppression of early opening of the shoulders can promote more favorable impact conditions. The NCHS was not associated with any X-factor stretch 
parameters (backswing, downswing, and total). It was apparent that normalization of the clubhead speed weakened the association between the X-factor/stretch parameters and clubhead speed (Table 2).

The $X$-factor range and stretch parameters were also characterized by significant deviations from normal distribution across all club conditions, suggesting swing style-dependent HL-SL torsional separations. Although not statistically significant, there was a tendency that higher NCHS was generally associated with larger backswing stretch than smaller downswing stretch (Table 2). Han and colleagues [2] reported that the LDS swing style, showing the largest downswing stretch among the styles, was characterized by a partial proximal-to-distal transition sequence with notable separation between the pelvis and the rest (HL $\rightarrow \mathrm{SL} / \mathrm{UL} / \mathrm{club})$. The LBS style, showing the largest backswing stretch, on the contrary, was characterized by a well-spaced, complete proximal-to-distal transition sequence (HL $\rightarrow \mathrm{SL} \rightarrow \mathrm{UL} \rightarrow \mathrm{club}$ ) with an elongated transition phase. While skilled male golfers overall showed proximal-to-distal transition sequences, the separation between the UL and club got obscured in the pitching wedge condition (Table 3). These findings suggest that more deviations from a full proximal-to-distal sequence may be observed in the shorter club conditions in some swing styles (e.g., LDS).

In a study involving 18 skilled male golfers, Kwon et al. [3] reported no significant correlation between the NCHS and the FSP-based X-factor/stretch parameters including the value at BI for the driver condition. The maximum X-factor and the downswing/total stretches reported in their study $\left(59^{\circ}\right.$, $1^{\circ}$, and $8^{\circ}$, respectively) were comparable to those from the current study $\left(61^{\circ}, 1^{\circ}\right.$, and $6^{\circ}$, respectively; Table 2). In a study involving a large heterogeneous group of golfers ( $n=100$, three ball speed groups), Myers et al. [28] reported significant correlations of ball speed to X-factor at TB $(r=0.55)$ and maximum $\mathrm{X}$-factor $(r=0.54)$. In another study involving an even larger heterogeneous group (308 golfers; 266 males and 42 females; $8.4 \pm 8.4$ handicap), Chu et al. [20] reported a significant regression that associated ball velocity with six kinematic parameters measured at TB: lateral bend, superior-inferior shift velocity, $\mathrm{X}$-factor, leading arm angle, wrist hinge, and leading knee flexion $\left(R^{2}=0.437\right)$. However, it is likely that these significant correlation/regression results were largely driven by the heterogeneity in the sample [35], evidenced by the reported significant inter-group differences in the ball speed and $X$-factor among various ball velocity [28] and skill [13] groups.

In terms of the kinematic-sequence time parameters, higher NCHS was associated with delayed onset of SL's backswing peak and delayed transitions of all bodies except SL (Table 3). The NCHS, however, was not associated with the timings of the downswing peaks in any consistent way. This lack of association in the downswing may be explained by the interplay of two conflicting necessities: (1) faster swing tends to delay the downswing peaks due to shorter downswing phase time; (2) the body has to reach peak angular velocities early enough to allow a sufficient time for the angular momentum transfer from the body to the club [2].

The backswing sequences were characterized by the separation between the angular velocity peaks of the axle and the chain (i.e., SL/HL vs. UL/club) (Table 3). The SL tended to reach the angular velocity peak earlier than the HL did in the backswing. Timings of the backswing peaks were characterized by large SDs when compared to the transition and downswing peak times due to the slower backswing motion and the large SDs in the backswing phase time (Tables 1 and 4). The transition sequences were more consistent than the other sequences, showing full or partial proximal-to-distal sequences (Table 3). Longer clubs (driver and 5-iron) exhibited full proximal-to-distal sequences while the pitching wedge condition lost the separation between the UL and club transition points. The timings of the downswing angular velocity peaks of the HL, SL, and UL were not well separated from each other and the sequences were not consistent across the club conditions (Table 3) [2]. The separation of the downswing peaks between the body (HL/SL/UL) and the club was obvious, but the peaks of the axle segments and the UL occurred within narrow time windows (14,13, and $9 \mathrm{~ms}$ on average for the driver, 5 -iron, and pitching wedge, respectively). In the driver condition, the angular velocity peaks of the axle segments (HL and SL) on average occurred at ED (Tables 1 and 3). As the club gets shorter, ED tends to occur earlier while the angular velocity peaks of the axle segments occur later (i.e., closer to BI). 
The peak angular velocities (downswing in particular) showed the strongest association with the NCHS (Table 4). Higher NCHS was associated with faster backward rotations of the body (HL, SL, and UL), but no association was observed with the angular speed of the club and the wrist. Club and wrist angular velocities during the backswing can easily be manipulated by the wrist action. While LB occurred on average $12 \mathrm{~ms}, 19 \mathrm{~ms}$, and $20 \mathrm{~ms}$ after LBA in the driver, 5-iron, and wedge conditions, respectively (Table 1), 23 (driver), 19 (5-iron), and 18 (pitching wedge) golfers (out of 66 each) actually showed the reversed sequence (LB $\rightarrow$ LBA). Early initiation of wrist cocking in the backswing using the wrist muscles causes the club and wrist to rotate faster/earlier and affects the way the body rotates. For example, Han and colleagues [2] reported that the STS (small total stretch) style exhibited a wrist-dominant swing pattern with early HL-SL separation and small X-factor stretches. It was speculated that the swing style-dependent variations weakened NCHS' association with the peak angular velocities of the club/wrist during the backswing.

Higher NCHS was also associated with faster downward rotations of all body parts of the axle-chain system, more slow-down of the HL and UL toward BI, and faster rotations of the SL and UL at BI (Table 4). Among the body parts, the UL revealed a unique correlation profile: i.e., the more it slows down after reaching the peak, and the faster the rotation still is at BI, the higher the NCHS is. The UL directly interacts with the club during the swing, and more slow-down in the UL rotation was found to be strongly associated with faster wrist uncocking $(r=0.758,0.770$, and 0.808 for the driver, iron, and pitching wedge, respectively). Effective slow-down of the UL rotation and speed-up of the wrist uncocking can be promoted with a delayed release [22].

Among the elements of the axle-chain system, the UL revealed significant correlations to NCHS across all club conditions in all four angular velocity parameter categories (with one exception of angular velocity at BI in the driver condition): backswing peak, downswing peak, velocity at BI, and peak-to-BI decrease. The SL showed significant correlations to clubhead speed in all categories except the peak-to-BI decrease. The HL showed significant correlations to clubhead speed in all categories except the angular velocity at BI.

From a supplementary correlation analysis, it was found in the HL and SL that the peak backswing angular velocity and the peak downswing angular velocity were correlated to each other significantly across all club conditions: $r=0.500,0.597$, and 0.591 for the driver, 5-iron, and pitching wedge, respectively, in the HL, and $r=0.339,0.493$, and 0.530 , respectively, in the SL. This means faster backward rotation of the axle tends to result in faster downward rotation of the axle. The backswing-downswing association got weaker in the UL and club/wrist, and the driver condition revealed weaker associations than the iron and wedge conditions did, suggesting added complexity in the UL/club/wrist motions, particularly in the driver swing.

The overall findings of this study (correlations and kinematic sequences) and those of Han and colleagues [1,2] collectively highlight the benefits of a faster backswing motion for skilled golfers. The potential benefits of active (faster) backswing include the following: (1) a faster backward rotation of the axle leads to a more body-driven backswing along with large backward angular momentums of the axle segments; (2) a body-driven backswing promotes the LBS swing pattern by increasing the backswing X-factor stretch and elongating the transition phase; (3) the continuous HL-SL torsional separation during the transition phase that comes with the LBS swing style induces full and well-spaced proximal-to-distal transition sequence; (4) a continuous proximal-to-distal transition promotes SSC-style muscle actions during the transition and early downswing phases making the swing easier with more efficient use of muscles; (5) an active and continuous backswing-to-downswing transition requires the lower body to interact with the ground more actively, resulting in large golfer-ground interaction moments (i.e., the GRF moment in the frontal plane and the pivoting moment in the horizontal plane) [1]; (6) large golfer-ground interaction moments at TB enable fast acceleration of the downward rotation of the body from the beginning of the downswing, thus shortening the early downswing phase without a waste of time; (7) early acceleration of the downward rotation allows the axle and the UL to reach their angular velocity peaks early and secure a sufficient time for the body-to-club angular 
momentum transfer; (8) the LBS-style (body-driven) swing also promotes delayed release of the club (i.e., wrist uncocking).

\section{Conclusions}

The main purpose of this study was to investigate the association between the on-plane angular motion parameters of the axle-chain system and the clubhead speed in three different club conditions (driver, 5-iron, and pitching wedge) in a group of skilled male golfers. It was concluded that:

- Higher normalized clubhead speed is associated with shorter downswing phases (total, early downswing, and late downswing) and more delayed onset of downswing events (TB and thereafter) closer to impact. In addition, a shorter early downswing phase is associated with a longer transition phase.

- Higher normalized clubhead speed is associated with larger backward/downward on-plane rotations (hip/shoulder lines and upper lever) on the functional swing plane. Normalized clubhead speed is not significantly associated with the wrist-cock angles or wrist motion ranges. In the driver swing, more wrist cocking at EDA (keeping the club closer to the body) is associated with higher normalized clubhead speed.

- Higher normalized clubhead speed is associated with a larger X-factor at BI (with the shoulder line trailing the hip line). Normalized clubhead speed is not significantly associated with other X-factor/stretch parameters.

- Higher normalized clubhead speed is associated with more delayed onset of backswing angular velocity peaks (shoulder line) and more delayed backswing-to-downswing transitions (hip/shoulder lines, upper lever, and club). Normalized clubhead speed is not significantly associated with the timings of the backswing angular velocity peaks.

- Only the transition sequence exhibits consistent proximal-to-distal sequences with a minor deviation in the pitching wedge condition. The backswing and downswing sequences deviate from the proximal-to-distal sequence significantly and are inconsistent across club conditions. The backswing sequence revealed a dominant separation between the axle (hip/shoulder lines) and the chain (upper lever and club), while the downswing sequence exhibited a dominant separation between the body and the club. The downswing angular velocity peaks of the body (hip/shoulder lines and upper lever) typically occur within a narrow time window.

- Higher normalized clubhead speed is associated with faster backward rotations (hip/shoulder lines and upper lever). Higher normalized clubhead speed is also associated with faster downward rotations (hip/shoulder lines and upper lever), more slowdowns after reaching the peak (hip line and upper lever), and faster rotations at BI (shoulder line and upper lever). Faster backward rotations are associated with faster downward rotations (hip/shoulder lines).

- While larger/faster backward and downward rotations of the axle-chain system on the functional swing plane are the overarching pre-requisites of a high clubhead speed, the correlation and kinematic sequence profiles fundamentally highlight two key aspects of a biomechanically robust golf swing: body-driven active (fast) backswing and active/elongated backswing-to-downswing transition.

Author Contributions: Conceptualization, M.V.M., K.A.B. and Y.-H.K.; methodology, all; software, Y.-H.K.; validation, M.V.M. and Y.-H.K.; formal analysis, M.V.M., M.A.A., N.A.L. and N.J.T.; investigation, M.V.M., M.A.A., N.A.L. and N.J.T.; resources, M.V.M. and Y.-H.K.; data curation, M.V.M., M.A.A., N.A.L. and N.J.T.; writing-original draft preparation, M.V.M. and Y.-H.K.; writing-review and editing, all; supervision, K.A.B. and Y.-H.K.; project administration, M.V.M. and Y.-H.K. All authors have read and agreed to the published version of the manuscript.

Funding: This research received no external funding.

Acknowledgments: The authors would like to thank Qualisys AB for providing an optical motion capture system.

Conflicts of Interest: The authors declare no conflict of interest. 


\section{References}

1. Han, K.H.; Como, C.; Kim, J.; Lee, S.; Kim, J.; Kim, D.K.; Kwon, Y.-H. Effects of the golfer-ground interaction on clubhead speed in skilled male golfers. Sports Biomech. 2019, 18, 115-134. [CrossRef]

2. Han, K.H.; Como, C.; Kim, J.; Hung, C.-J.; Hasan, M.; Kwon, Y.-H. Effects of pelvis-shoulders torsional separation style on kinematic sequence in golf driving. Sports Biomech. 2019, 18, 663-685. [CrossRef]

3. Kwon, Y.-H.; Han, K.H.; Como, C.; Lee, S.; Singhal, K. Validity of the X-factor computation methods and relationship between the $\mathrm{X}$-factor parameters and clubhead velocity in skilled golfers. Sports Biomech. 2013, 12, 231-246. [CrossRef]

4. Kwon, Y.-H.; Como, C.S.; Han, K.; Lee, S.; Singhal, K. Assessment of planarity of the golf swing based on the functional swing plane of the clubhead and motion planes of the body points. Sports Biomech. 2012, 11, 127-148. [CrossRef]

5. Putnam, C.A. Sequential motions of body segments in striking and throwing skills: Descriptions and explanations. J. Biomech. 1993, 26, 125-135. [CrossRef]

6. Marshall, R.N.; Elliott, B.C. Long-axis rotation: The missing link in proximal-to-distal segmental sequencing. J. Sports Sci. 2000, 18, 247-254. [CrossRef]

7. Fuchs, P.X.; Lindinger, S.J.; Schwameder, H. Kinematic analysis of proximal-to-distal and simultaneous motion sequencing of straight punches. Sports Biomech. 2018, 17, 512-530. [CrossRef]

8. Wagner, H.; Pfusterschmied, J.; Duvillard, S.P.V.; Müller, E. Skill-dependent proximal-to-distal sequence in team-handball throwing. J. Sports Sci. 2012, 30, 21-29. [CrossRef]

9. Hellstrom, J. Competitive golf: A review of the relationships between playing results, technique and physique. Sports Med. 2009, 39, 723-741. [CrossRef]

10. Joyce, C.; Burnett, A.; Cochrane, J.; Ball, K. Three-dimensional trunk kinematics in golf: Between-club differences and relationships to clubhead speed. Sports Biomech. 2013, 12, 108-120. [CrossRef]

11. Lynn, S.K.; Frazier, B.S.; New, K.N.; Wu, W.F.W.; Cheetham, P.J.; Noffal, G.J. Rotational kinematics of the pelvis during the golf swing: Skill level differences and relationship to club and ball impact conditions. Int. J. Golf Sci. 2013, 2, 116-125. [CrossRef]

12. Vena, A.; Budney, D.; Forest, T.; Carey, J. Three-dimensional kinematic analysis of the golf swing using instantaneous screw axis theory, Part 2: Golf swing kinematic sequence. Sports Eng. 2011, 13, 125-133. [CrossRef]

13. Zheng, N.; Barrentine, S.W.; Fleisig, G.S.; Andrews, J.R. Kinematic analysis of swing in pro and amateur golfers. Int. J. Sports Med. 2008, 29, 487-493. [CrossRef] [PubMed]

14. Cheetham, P.J.; Rose, G.A.; Hinrichs, R.N.; Neal, R.J.; Mottram, R.E.; Hurrion, P.D.; Vint, P.F. Comparison of kinematic sequence parameters between amateur and professional golfers. In Science and Golf V: Proceedings of the World Scientific Congress of Golf; LPGA Foundation: Phoenix, AZ, USA, 2008.

15. Horan, S.A.; Kavanagh, J.J. The control of upper body segment speed and velocity during the golf swing. Sports Biomech. 2012, 11, 165-174. [CrossRef] [PubMed]

16. Tinmark, F.; Hellström, J.; Halvorsen, K.; Thorstensson, A. Elite golfers' kinematic sequence in full-swing and partial-swing shots. Sports Biomech. 2010, 9, 236-244. [CrossRef]

17. Burden, A.M.; Grimshaw, P.N.; Wallace, E.S. Hip and shoulder rotations during the golf swing of sub-10 handicap players. J. Sports Sci. 1998, 16, 165-176. [CrossRef]

18. Okuda, I.; Gribble, P.; Armstrong, C. Trunk rotation and weight transfer patterns between skilled and low skilled golfers. J. Sports Sci. Med. 2010, 9, 127-133.

19. Egret, C.; Dujardin, F.; Weber, J.; Chollet, D. 3-D kinematic analysis of the golf swings of expert and experienced golfers. J. Hum. Motion Mov. Stud. 2004, 47, 193-204.

20. Chu, Y.; Sell, T.C.; Lephart, S.M. The relationship between biomechanical variables and driving performance during the golf swing. J. Sports Sci. 2010, 28, 1251-1259. [CrossRef]

21. Nesbit, S.M. A three dimensional kinematic and kinetic study of the golf swing. J. Sports Sci. Med. 2005, 4, 499-519.

22. Sprigings, E.J.; Mackenzie, S.J. Examining the delayed release in the golf swing using computer simulation. Sports Eng. 2002, 5, 23-32. [CrossRef]

23. Hume, P.A.; Keogh, J.; Reid, D. The role of biomechanics in maximising distance and accuracy of golf shots. Sports Med. 2005, 35, 429-449. [CrossRef] [PubMed] 
24. Cheetham, P.J.; Martin, P.E.; Mottram, R.E.; St. Laurent, B.F. The importance of stretching the 'X-Factor' in the downswing of golf: The 'X-Factor stretch'. In Optimising Performance in Golf; Thomas, P.R., Ed.; Australian Academic Press: Brisbane, QLD, Australia, 2001; pp. 192-199.

25. McLean, J. Widen the gap. Golf Mag. 1992, 1992, 49-53.

26. Cole, M.; Grimshaw, P. The X-factor and its relationship to golfing performance. J. Quant. Anal. Sports $2009,5$. [CrossRef]

27. McTeigue, M.; Lamb, S.R.; Mottram, R.; Pirozzolo, F. Spine and hip motion analysis during the golf swing. In Science and Golf II: Proceedings of the World Scientific Congress of Golf; Cochran, A.J., Farrally, M.R., Eds.; E \& FN Spon: London, UK, 1994; pp. 50-58.

28. Myers, J.; Lephart, S.; Tsai, Y.S.; Sell, T.; Smoliga, J.; Jolly, J. The role of upper torso and pelvis rotation in driving performance during the golf swing. J. Sports Sci. 2008, 26, 181-188. [CrossRef]

29. Horan, S.A.; Evans, K.; Morris, M.R.; Kavanagh, J.J. Thorax and pelvis kinematics during the downswing of male and female skilled golfers. J. Biomech. 2010, 43, 1456-1462. [CrossRef]

30. Meister, D.W.; Ladd, A.L.; Butler, E.E.; Zhao, B.; Rogers, A.P.; Ray, C.J.; Rose, J. Rotational biomechanics of the elite golf swing: Benchmarks for amateurs. J. Appl. Biomech. 2011, 27, 242-251. [CrossRef]

31. Lephart, S.M.; Smoliga, J.M.; Myers, J.B.; Sell, T.C.; Tsai, Y.S. An eight-week golf-specific exercise program improves physical characteristics, swing mechanics, and golf performance in recreational golfers. J. Strength Cond. Res. 2007, 21, 860-869. [CrossRef]

32. Winter, D.A. Biomechanics and Motor Control of Human Movement, 3rd ed.; John Wiley \& Sons: Hoboken, NJ, USA, 2005.

33. Bell, A.L.; Pedersen, D.R.; Brand, R.A. A comparison of the accuracy of several hip center location prediction methods. J. Biomech. 1990, 23, 617-621. [CrossRef]

34. Lee, D.K. Alternatives to P value: Confidence interval and effect size. Korean J. Anesthesiol. 2016, 69, 555-562. [CrossRef]

35. Ball, K.A.; Best, R.J. Different centre of pressure patterns within the golf stroke I: Cluster analysis. J. Sports Sci. 2007, 25, 757-770. [CrossRef] [PubMed]

(C) 2020 by the authors. Licensee MDPI, Basel, Switzerland. This article is an open access article distributed under the terms and conditions of the Creative Commons Attribution (CC BY) license (http://creativecommons.org/licenses/by/4.0/). 